\title{
A computer-controlled laser interferometer
}

\author{
D. W. HEELEY \\ Bell Laboratories, Murray Hill, New Jersey 07974
}

\begin{abstract}
An apparatus is described for generating sinusoidal interference fringes on the retina of the eye under computer control. The novel aspect of the design is the use of acoustooptic modulators to pulse-width modulate the laser beams. This gives flexible control of the contrast of the fringes.
\end{abstract}

Sinusoidal grating stimuli have become ubiquitous in the study of spatial contrast vision (e.g., Blakemore \& Campbell, 1969). Virtually all oscilloscope displays that employ television techniques are limited in their application if high brightness levels or high contrasts are required. They are also confined to a limited range of temporal modulations.

The eye optics also affect the retinal image and, due to the diffraction limit, severely attenuate the contrast at high spatial frequencies (e.g., Krauskopf, 1962). Differential accommodative responses to different frequency targets compound this problem.

An alternative approach is to exploit the wave nature of light to produce interference fringes directly on the retina (e.g., Campbell \& Green, 1965; Westheimer, 1960). This may be more easily accomplished if the source is coherent. An expanded beam of laser light can be split into two paths of different lengths, and these then can be combined and focused in the eye. They then expand to form two overlapping circular patches, with interference fringes of sinusoidal profile filling the region of overlap. The advantage is that the spacing and contrast of the fringes is almost completely independent of the refractive state of the eye. If the energy of the two beams is exactly balanced, the contrast of the fringes will be 1.0. The spacing of the fringes is a linear function of the separation of the effective "point" sources created by focusing the beams: $\alpha=\lambda / D$, where $\alpha$ is the distance between successive maxima in radians, $\lambda$ is the wavelength of the laser light, and D is the separation between the "points."

A particularly versatile instrument for producing such interference patterns is one based on the MachZehnder interferometer (Born \& Wolf, 1975). An elegant modification of this has been previously described (Burton, 1973) that enables the spatial frequency of the fringes to be rapidly and accurately altered. The geometrical theory of this modification is described in detail in Burton's paper.

In any interferometer, the contrast of the fringes

I gratefully acknowledge the encouragement and support of Dr. J. Krauskopf during the design of this apparatus and his critical reading of a draft of this note. Dr. Krauskopf designed and constructed the electronic circuits to drive the modulators. may be altered from a value of 1.0 by varying the relative intensity of the beams in the two arms of the instrument or by adding a desaturating field of coherent or incoherent light to the fringes. This is usually achieved by the use of a rotating polaroid or by the use of neutral density filters. Neither of these techniques lends itself particularly well to computer control, and neither can be easily employed to generate an arbitrarily complex temporal modulation of contrast (such as a Gaussian).

The present apparatus uses acoustooptic modulators to achieve this control. It is illustrated in Figure 1. Two low-power $\mathrm{He} / \mathrm{Ne}$ lasers are fitted with commercially available acoustooptic modulators (Coherent Associates Model 305). These modulators comprise mounted rectangular glass prisms to which have been cemented ultrasonic transducers. The piezoelectric transducers set up high-frequency standing waves within the glass, which diffract an incident laser beam (Dixon, 1971). The emergent pattern of light thus comprises an undeflected beam that has passed directly through the prism, plus a set of different diffraction orders. The modulator may be adjusted so that $50 \%$ of the main beam energy can be deflected into one of the first diffraction orders under ideal conditions. The angular deviation of the first-order diffracted beam is approximately $.7 \mathrm{deg}$. The lasers are thus pointed slightly upward so that the diffracted beam

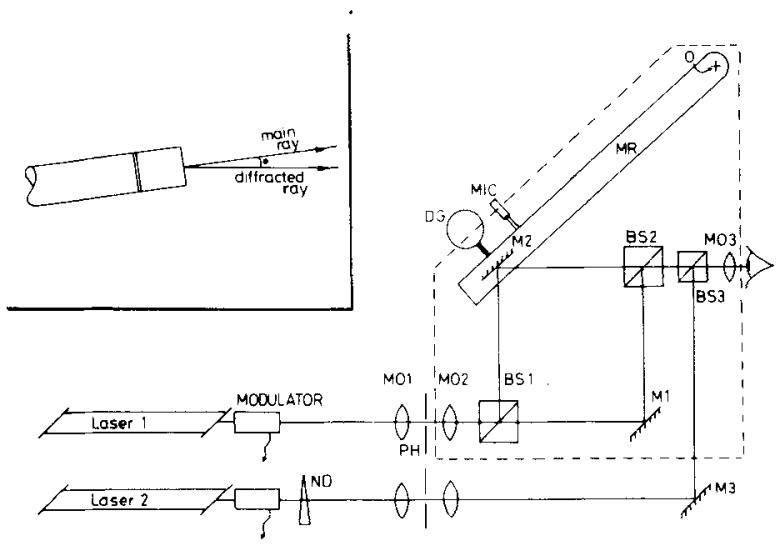

Figure 1. Plan view of the interferometer. The inset shows the tilted laser/modulator combination. The angle $\theta$ has been greatly exaggerated. 
travels on a path that is parallel to the optic bench on which the apparatus is mounted (see inset in Figure 1).

These beams are then focused by microscope objective $\mathrm{MOl}$ onto a 25 -micron pinhole, $\mathrm{PH}$. This serves the multiple purpose of (1) excluding the main beam from the apparatus, (2) ensuring the selection of a single laser mode $\left(\mathrm{TEM}_{00}\right)$, and (3) providing some spatial filtering of the wave-front defects caused by the passage of the laser beam through the laser optics and modulator (Lipson, 1972). The emergent cone of rays is then collimated and expanded by the low-power objective $\mathrm{MO} 2$ and split into two paths in the interferometer by the beam splitter cube, BS1. Following deflection by mirrors $M 1$ and $M 2$, the two beams are recombined at beam splitter BS2 (closely matched to BS1) and then brought to a focus in the eye by the objective MO3 serving as an eyepiece. Mirrors M1 and $\mathrm{M} 2$ are first-surface Pyrex, flat to $1 / 10$ wave. Contrast is controlled by the introduction of the light from a second laser, which is combined as a desaturating field by beam splitter cube BS3. Identical spatial filtering is employed, and a neutral density wedge, ND, is inserted in the beam such that relative intensity of the two sources can be balanced.

The acoustooptic modulators allow either analog or digital control. Digital control permits much better extinction ratios than are attainable with analog techniques, and it is ideally suited to a digital computation approach. With pulse-width modulation, this results in extremely precise and flexible control of intensity as long as the repetition frequency is substantially less than the integration time of the eye. The visual system cannot resolve the $3-\mathrm{msec}$ flicker, the repetition rate used in the present apparatus, integrating the light from the background desaturant and fringe sources, resulting in fringes of a contrast dependent on the relative durations of the two sources. Any decrease in the duty cycle of the laser generating the fringes is compensated for by a concomittant increase in the duration of the pulse width of the background laser beam. This ensures that the mean luminance of the display remains constant.

In the present implementation of the device, the spatial frequency of the fringes is altered manually by advancing or retarding the micrometer screw, MIC. This results in a radial movement of arm MR bearing mirror M2 and, hence, changes the spacing of the fringes. Over a limited range of travel, the change in the spacing of the fringes is a linear function of the distance by which the micrometer is moved. This is measured to a high degree of accuracy by the dial guage, DG. The spatial frequency of the fringes may also be altered by changing the power of the eyepiece. More complete computer control can be achieved by fitting the micrometer screw with a stepping motor.

Those components shown within the broken line in Figure 1 are mounted on a metal plate $5 / 8$ in. thick.
This is attached to the optical bench carrying the remainder of the optical system. The subject's head is restrained by a dental bite mounted on a carrier on the transverse optical bench running underneath the interferometer. Attention to the mechanical details of construction is required, as vibration displaces the fringes on the retina and reduces their apparent contrast.

\section{PHASE-REVERSING GRATINGS}

The interferometer may be easily modified to produce fringes whose contrast can be modulated from -1.0 to +1.0 . The modifications required are shown in Figure 2.

Beam splitter cube BS2 is removed, and mirror M3 is placed at the position shown. This introduces the filtered, collimated light from Laser 2 into the interferometer on a path orthogonal to that of Laser 1. This then creates a second set of fringes of contrast 1.0 and of the same frequency and in the same retinal location as those due to the primary laser source. A phase plate, $\mathrm{PP}$, displaces the primary fringes by $180 \mathrm{deg}$ to exactly cancel those from Laser 2. Compensated modulation of the two lasers thus results in fringes whose contrast may be set at any level in the range -1.0 to +1.0 . Again, any arbitrarily complex temporal contrast function can be utilized. This technique is not quite as successful as the desaturating field. Minor inhomogeneities in the beam splitter cubes create small areas of the field that fail to cancel. A more critical choice of high-quality optical elements will probably overcome this defect.

\section{CONTROL CIRCUIT AND INTERFACE}

The 40-MHz RF oscillators and amplifiers required to drive the piezoelectric elements inside the modulators are supplied by the manufacturers (Coherent Associates Model 305D). They may be gated on and off by apply. ing a TTL signal to the blanking input of the amplifier. These trigger pulses are derived from the circuit shown in schematic form in Figure 3.

Three 16-bit data words are multiplexed on a data

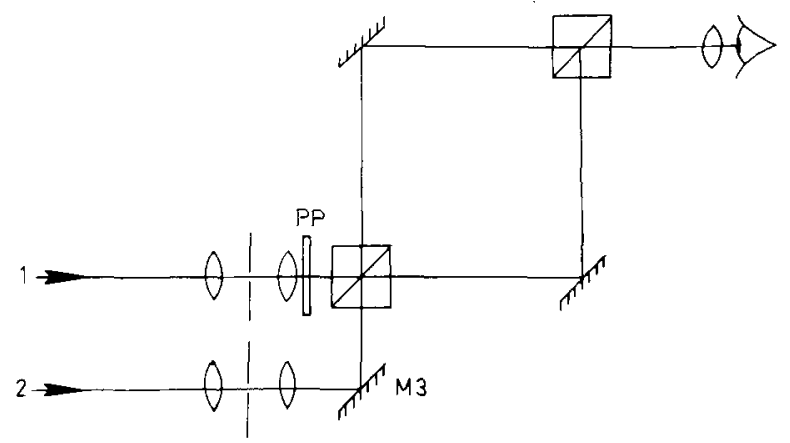

Figure 2. Modified version of the interferometer to provide phase reversal. 


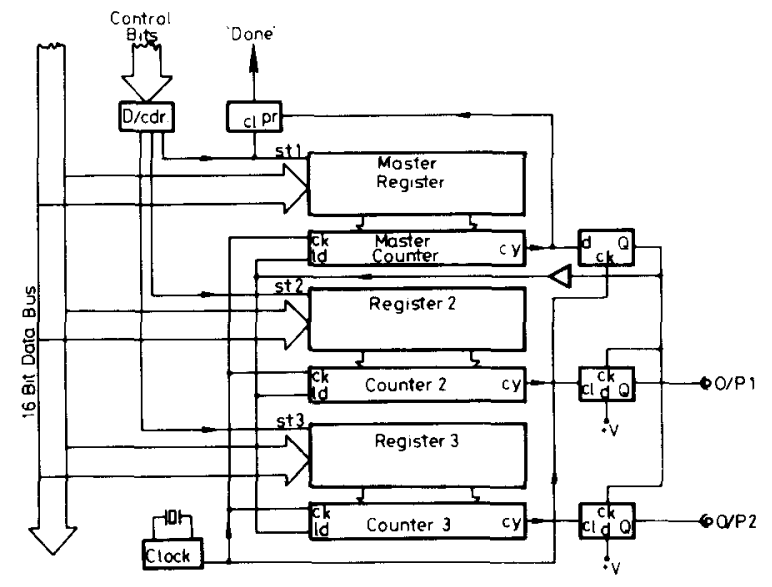

Figure 3. Schematic diagram of the electronic circuit providing the variable-width pulses; part of the interface is also shown. For full description, see text.

bus from a standard laboratory computer interface. These words are used to define the duration of the main cycle and the durations of the pulses output to Lasers 1 and 2. Three additional control bits are decoded (D/CDR, LS138) and steer the data into the control registers (LS174) by setting the relevant strobe lines (st $1, \mathrm{st} 2, \mathrm{st} 3)$.

The registers load three identical programmable counters (LS193) that are clocked synchronously at approximately $10 \mathrm{MHz}$ by a crystal-locked oscillator (LS124). The trigger signals (O/Ps 1 and 2) to the RF amplifiers are derived from the output (Q) of two D-type flip-flops (LS74). Initially, these outputs are at logic high as the data inputs (d) are connected to the positive power supply rail. However, when either Counter 2 or 3 completes its respective cycle, the flipflops are cleared by applying the carry bit (cy) of the counter to the clear (cl) input of the flip-flop.

When the master counter completes its count cycle, the carry bit is clocked through a similar flip-flop on the next clock pulse, and the pulse thus derived resets the output flip-flops via the clock (ck) input. The carry bit from the master counter is also buffered and used to reload the counters from the data registers. A new counting cycle then automatically commences.

To indicate successful loading of the data registers, a "done" signal is passed back to the computer. The master count carry bit presets a flip-flop at the end of the first count cycle. This is only cleared when the strobe line, st1, goes logic high to indicate that a fresh data sequence is about to be passed down the data bus. Once loaded, the counter circuit operates completely independently of the computer, maintaining the data values in the registers until they are overwritten.

At present, the modulator circuit is incorporated in a laboratory interface connected to a PDP.11/03 computer. The data registers have hard-wired addresses and can be loaded by direct addressing from any of the languages supported by the computer. The apparatus described is currently in use in the study of contrast increment thresholds on subthreshold and suprathreshold backgrounds. It is found to be easy to use, and it yields reliable and replicable results. The fringes produced are strictly sinusoidal, without harmonic distortion, and they may be of exceptionally high brightness and contrast. They may also be of a spatial frequency that considerably exceeds that attainable with conventional large-field oscilloscope-type displays.

\section{REFERENCES}

Blakemore, C. B., \& Campbell, F. W. On the existence of neurones in the human visual system selectively sensitive to the orientation and size of retinal images. Journal of Physiology, London, 1969, 203, 237-260.

Bonn, M., \& Wolf, E. Principles of optics (5th ed.). Oxford: Pergamon Press, 1975.

Bunton, G. J. Evidence for non-linear response processes in the human visual system from measurements on the thresholds of spatial beat frequencies. Vision Research, 1973, 13, 1211-1225.

Campbell, F. W. C., \& Green, D. G. Optical and retinal factors affecting visual resolution. Journal of Physiology, London, 1965, 181, 576-593.

Dixon, R. W. The acoustooptic interaction. In W. A. Albers (Ed.), The physics of opto-electronic materials. New York: Plenum, 1971.

KrausKopf, J. Light distribution in human retinal images. Journal of the Optical Society of America, 1962, 52, 1046-1050.

Lipson, H. Optical transforms. New York: Academic Press, 1972.

Westheimer, G. Modulation thresholds for sinusoidal light distributions on the retina. Journal of Physiology, London, $1960,152,64-67$.

(Received for publication June 27, 1979; revision accepted July 20,1979 .) 